\title{
¿Es el argumento del conocimiento de Jackson un knock-out al fisicalismo reductivo?
}

doi: 10.33264/rpa.201901-04

\author{
Agustín Fernando Lavoz Torres \\ Docente Jornada, Área de Coaching \\ Docente titular del Magíster en Coaching Organizacional \\ Facultad de Administración, UNIACC
}

\section{Resumen}

El presente artículo busca responder la pregunta sobre si el argumento del conocimiento planteado por Frank Jackson, en los casos de Mary y Fred, logra ser una crítica demoledora en contra del fisicalismo reductivista. El argumento del conocimiento de Jackson tuvo como objetivo probar la insuficiencia del fisicalismo reductivista para explicar la realidad, como también demostrar la existencia de qualia epifenoménicos (elementos no físicos e impotentes causalmente). Mi respuesta a la pregunta planteada es negativa, puesto que considero que el fisicalismo reductivista sí logra superar el problema planteado por Jackson valiéndose de sus propias premisas y que, por otra parte, los qualia epifenoménicos sí poseen un cierto rol causal.

Palabras clave: fisicalismo, qualia, causación mental, epistemología, ontología.

\section{Abstract}

This article seeks to answer the question of whether the knowledge argument raised by Frank Jackson, in the cases of Mary and Fred, manages to be a devastating criticism about reductivist physicalism. The aim of Jackson's knowledge argument was to prove the insufficiency of reductivist physicalism to explain reality, as well as to demonstrate the existence of epiphenomenal qualia (non-physical and impotent elements causally). My answer to the question is negative, since I consider that reductivist physicalism manages to overcome the problem posed by Jackson using himself and, on the other hand, I consider that epiphenomenal qualia does have a certain causal role.

Keywords: physicalism, qualia, mental causation, epistemology, ontology. 


\section{Introducción}

El término fisicalismo (physicalism) aparece en la filosofía durante la primera mitad del siglo XX en el marco del Círculo de Viena (Wiener Kreis), un proyecto investigativo basado en el empirismo, el método inductivo, la abolición de la metafísica y el establecimiento de un lenguaje común para la ciencia. Pese a que fueron Rudolph Carnap y Otto Neurath quienes introducen el término en la filosofía en los años de 1930 (Stoljar, 2010, p. 10), lo hacen en el marco de la filosofía del lenguaje, de modo que sobre ellos podemos mencionar que postulan un fisicalismo lingüístico. Posteriormente, la discusión acerca de la naturaleza (estatuto ontológico) del mundo se focaliza en la discusión acerca de la naturaleza del cuerpo y de la mente. Esta discusión es tan antigua como lo es la filosofía: pensadores como Platón o Descartes, sostienen que la mente y cuerpo son entidades separadas y, además, entidades de distinta naturaleza, donde el cuerpo es una entidad extensa (que ocupa un espacio y tiene características contingentes) y la mente es una entidad inmaterial (o pensable, como sostuvo Descartes). A esta forma de pensar se le ha denominado dualismo sustancial. Por otro lado, filósofos como Epicuro (pensador atomista del siglo IV y III a.C.) o algunos más contemporáneos como U.T. Place sostienen que mente y cuerpo en realidad son una misma entidad o, al menos, dos entidades de la misma naturaleza extensa, corpórea o física. A esta forma de pensar se le denomina, mutatis mutandi, como fisicalismo. Entre estas dos grandes posturas extremas hay una gran variedad de propuestas intermedias: fisicalismos más débiles o dualismos más débiles.

El fisicalismo reductivista es la variante más extrema del fisicalismo, y sostiene que todo es físico y, por lo tanto, que todo lo que pensamos, vemos o aprendemos es reductible a lo físico. De modo que toda propiedad o evento mental $\mathrm{M}$ es reductible a alguna propiedad o evento físico P. Por ejemplo, Place (1956) sostiene que los procesos mentales se identifican con (es decir, son) procesos fisico-químicos del cebrero. Una de las principales críticas al fisicalismo en las últimas décadas del siglo XX fue el así llamado argumento del conocimiento de Frank Jackson (1982, 2003) y $(1986,2003)$. Si bien no es un argumento totalmente original de Jackson ${ }^{4}$, fue él quien le otorgó su formulación clásica mediante los casos de Fred (quien experimenta un color que ninguno de nosotros ve, denominado como rojo ${ }_{2}$ ) y de Mary (una científica brillante del futuro quien experimenta por primera vez el color, tras estar confinada a un cuarto en blanco y negro). Esto abrió el espacio para amplios debates que se dieron principalmente durante la década de 1980 y que se mantiene, aunque en

\footnotetext{
${ }^{4}$ Para una síntesis de formulaciones anteriores a las de Jackson ver (Alter, 2005).
} 
menor medida, vigente en la actualidad5. El argumento de Jackson afirma que el fisicalismo (como conocimiento total de lo físico) es insuficiente, y que es válida la existencia de cualidades sensoriales (qualia) no-físicas y causalmente impotentes (epifenoménicas) ${ }^{6}$.

En este escrito quiero resolver la siguiente pregunta, a saber: ¿Constituye el argumento del conocimiento de Frank Jackson una crítica demoledora al fisicalismo reductivo? Mi respuesta es que no. Considero que el fisicalismo reductivo (que todo M (evento o propiedad mental) es reductible a $\mathrm{P}$ (evento o propiedad física)) logra superar el problema planteado por Jackson valiéndose de sus propias premisas; no obstante, para responder a este problema debe asumirse, a mi juicio, la existencia de qualia sensoriales físicos ${ }^{7}$ y concederles, al menos en parte, un cierto rol causal ${ }^{8}$.

\section{El argumento del conocimiento de Jackson}

Jackson presenta dos casos relacionados con la percepción visual de los colores, aunque extiende su argumento para los demás sentidos (Jackson, 1982, 2003, p. 100). Estos casos corresponden a Fred quien, al menos, percibe un color adicional al de nuestra gama de colores, y el caso de Mary, una súper científica del futuro.

\section{a) El caso de Fred.}

Fred tiene la mejor visión de colores que cualquiera en el mundo, hace clasificaciones de colores que ninguno de nosotros sería capaz de realizar:

Si se le muestra un montón de tomates maduros, los clasificará en dos grupos aproximadamente iguales y lo hará con absoluta consistencia. Es decir, se le vendan los ojos, se mezclan los tomates y luego se le retira la venda y se le pide que los clasifique de nuevo, los clasificará exactamente en los mismos dos grupos. (Jackson, 1982, 2003, p. 97)

\footnotetext{
5 Cabe mencionar que Jackson finalmente se retractó de su argumento inicial al apoyar un fisicalismo representacionalista (Jackson, Postscript on qualia, 1998).

6 Los qualia son la piedra en el zapato para el fisicalismo. Para Kim (2005, p. 170) son el "residuo mental que no puede acomodarse al dominio físico". Un quale (singular de qualia) es una cualidad sensorial: por ejemplo, cuando observamos un objeto rojo, el matiz de rojo que cada uno puede percibir es distinto para otro sujeto. De esta manera, es una cualidad sensorial dependiente del observador. Esta cualidad no tiene un sustrato físico propiamente tal. Esto también puede verse en el caso del dolor: el cómo es percibido un mismo estímulo doloroso varía entre cada sujeto.

${ }^{7}$ A mi juicio, la única forma de sostener la existencia de los qualia, desde el fisicalismo reductivo, es atribuirles una ontología física (considerando que el fisicalismo asumido totalmente no puede considerar un dominio no- físico).

${ }^{8}$ Esta es la respuesta de Kim (2005): los qualia no son totalmente impotentes, desde el punto de vista causal.
} 
Ciertamente, estos dos grupos de tomates maduros son para nosotros exactamente de un mismo color, al cual llamamos rojo. No obstante, para Fred, uno de aquellos grupos es equivalente a nuestra sensación de rojo y el otro grupo es de otro color radicalmente distinto (tanto como para nosotros es la diferencia entre azul y amarillo). Sin embargo, Fred ha intentado, por todos los medios, hacernos saber que existe dicha diferencia tan radical y que, por nuestra limitación visual, somos incapaces de percibir e incluso aceptar como verdaderos. Para ajustarse a nuestra limitación visual, Fred ha determinado catalogar el primer grupo de tomates maduros como rojo 1 y al otro como rojo ${ }_{2}$, siendo este último el cual no podemos percibir. "De cualquier modo, nos explica que sería bastante equivocado pensar que porque „rojo" aparece tanto en ",rojo ${ }^{\prime \prime}$ como en ",rojo ${ }_{2}$ ", ambos colores son tonos de un mismo color." (Jackson, 1982, 2003, p. 97) El sistema visual de Fred ha sido estudiado profundamente por neurofisiólogos, quienes han determinado que "[...] es capaz de separar dos grupos de longitudes de onda en el espectro del rojo con tanta nitidez como los demás son capaces de distinguir el amarillo del azul." (Jackson, 1982, 2003, p. 98).

¿Qué experiencia tiene Fred cuando ve rojo y rojo ${ }_{2}$ ? Nosotros, incapaces de ver el $\mathrm{rojO}_{2}$, deseamos saber qué experimenta Fred cuando percibe dicho color: ¿Puede la ciencia física dar cuenta de esta experiencia?, ¿Se puede conocer la experiencia que tiene Fred al experimentar rojo ${ }_{2}$ apelando a explicaciones de la ciencia reductiva? La respuesta de Jackson es negativa: si la neurofisiología completa ${ }^{9}$ conocía todo acerca del sistema visual de Fred, pero no puede explicar la experiencia de ver rojo $_{2}$ que tiene Fred para que, por ejemplo, nosotros podamos saber lo que experimenta Fred al ver rojo $_{2}$, entonces la neurofisiología y la ciencia física están dejando algo fuera de explicación. Es algo que no pueden explicar, de modo que el fisicalismo es falso. Incluso si, por algún invento notable de la ciencia del futuro, logramos implantar un chip en nuestro cerebro que logre hacernos experimentar lo que Fred experimenta al ver $\mathrm{rojO}_{2}$ y nos hace capaces, también, de clasificar los dos grupos de tomates que discriminaba Fred, entonces aprendemos y, por ende, ahora sabemos lo que es ver rojo $_{2}$, de modo que el fisicalismo el falso.

\footnotetext{
${ }^{9}$ Una ciencia completa es un estado ideal. Corresponde a una disciplina que ha logrado conocer todo objeto capaz de ser conocido por dicha ciencia. De esta manera, una neurofisiología completa ha accedido a todos los objetos propios de su saber y ya no queda nada más por descubrir.
} 


\section{b) El caso de Mary.}

El ejemplo de $\operatorname{Mary}^{10}$, el más popular en la discusión en torno al argumento del conocimiento, nos propone imaginar una científica brillante del futuro, Mary, quien tiene un conocimiento acabado de la física y la neurofisiología de la visión, pero con una particularidad: "[...] ha sido forzada a investigar el mundo desde una habitación en blanco y negro, mediante un monitor de televisión en blanco y negro." (Jackson, 1982, 2003, p. 100) De modo que jamás ha experimentado color alguno, salvo el blanco, negro y tonalidades de gris; no obstante, conoce todo acerca de la física de los colores: "Descubre, por ejemplo, precisamente qué combinaciones de longitudes de onda del cielo estimulan la retina [...]" (Jackson, 1982, 2003, p. 100) De modo que:

Conoce todos los hechos físicos acerca de nosotros y nuestro entorno, en un sentido amplio de "físico" que incluye todo lo que hay según la física, la química y la neurofisiología acabadas, y todo lo que hay que saber acerca de los hechos causales y relacionales resultado de todo esto, incluyendo, por su puesto, los papeles funcionales (Jackson, 1986, 2003, p. 111)

Pero Mary, quien nunca había experimentado color alguno (salvo el blanco, negro y tonalidades de gris) es liberada de su habitación (o bien se le entrega un monitor de televisión a color). ¿Hace alguna diferencia el que Mary ahora experimente los colores de los cuales sabía todo lo que había por saber desde la física y la neurofisiología del color? En rigor la pregunta es: ¿Aprende Mary algo nuevo al experimentar, por ejemplo, por primera vez el rojo de un tomate maduro?, en otras palabras, ¿se añade algo nuevo a su conocimiento previo sobre los colores? "Parece perfectamente obvio que aprenderá algo acerca del mundo y de la experiencia visual que tenemos de él." (Jackson, 1982, 2003, p. 100) Según Jackson, ella aprenderá cómo es ver algo rojo (1986, 2003, p. 112). ${ }^{11}$

El conflicto para el fisicalismo, según Jackson, es el siguiente: si el fisicalismo es verdadero y, por tanto, el hecho de que Mary conocía, antes de ser liberada de su habitación, toda la información física posible (en una ciencia física y neurofisiología acabadas), y ahora aprende algo nuevo (el cómo es ver el rojo), entonces dicha información física total y acabada dejaba algo fuera de la explicación: algo que, por

\footnotetext{
${ }^{10}$ Que, a diferencia de Fred, es una persona absolutamente normal o, al menos, igual a nosotros en su constitución neurofisiológica.

${ }^{11}$ Un fisicalista aceptaría esta conclusión; por ejemplo, Papineau (2002).
} 
no entrar en ella, resulta ser de distinta naturaleza. Lo que Mary aprende (el cómo es ver rojo) no puede ser algo físico, de modo que el fisicalismo es falso.

\section{c) Qualia epifenoménicos no-físicos (lo que Mary no sabía).}

Lo que Mary no conocía era el quale de color al percibir el rojo de un tomate maduro (y todos los demás qualia de colores del mundo). "La conclusión en cada caso es que los qualia quedan fuera de la explicación fisicalista." (Jackson, 1982, 2003, p. 100) De modo que estos rasgos fenoménicos o sensación cruda, como los denomina Jackson, no son físicos: no obstante, esto no es todo. Aun siendo no-físicos, por no entrar en la explicación fisicalista del mundo, los qualia también son impotentes causalmente, es decir, no cumplen ningún rol que afecte al mundo físico: “[...] es posible que ciertas propiedades de ciertos estados mentales, a saber, aquellas a las que he llamado qualia, son tales que su posesión o su ausencia no constituye ninguna diferencia para el mundo físico." (Jackson, 1982, 2003, p. 104) 12 $^{12}$

\section{Algunas respuestas fisicalistas al problema}

En esta sección quiero exponer algunas respuestas por parte del fisicalismo al problema presentado por el argumento del conocimiento de Jackson. La primera corresponde a Paul Churchland (1985), particularmente en su primera objeción: la ambigüedad del sentido de saber acerca de (know about); la segunda, corresponde a Terence Horgan (1984), según la cual el argumento del conocimiento de Jackson constituye una falacia al equivocarse sobre dos sentidos distintos de información física; por último, me referiré a la respuesta de David Papineau. Este último utiliza el argumento del conocimiento de Jackson para ejemplificar su propia teoría del monismo sustancial y dualismo conceptual. ${ }^{13}$

\section{a) Churchland: el problema del know about. ${ }^{14}$}

Churchland reconstruye el argumento de Mary de la siguiente manera:

(1) Mary sabe todo lo que hay que saber acerca de (to know about) los estados del cerebro y sus propiedades.

\footnotetext{
${ }^{12}$ Agregaré algunas consideraciones sobre esta temática más adelante en este escrito.

13 Los qualia serían, según Papineau (2002, p. 47) conceptos fenoménicos que refieren a sustancias materiales (únicas existentes).

14 En (1986, 2003) Jackson responde a las tres objeciones que Churchland (1985) realiza contra su argumento. La primera de estas es la que evaluaremos en esta sección; no obstante, Churchland (1989) responde ante la réplica de Jackson, sosteniendo que el error cometido en 1982 sobre el "know about” persiste en el artículo de 1986).
} 
(2) Este no es el caso en que Mary sabe todo lo que hay que saber acerca de las sensaciones y sus propiedades.

Entonces, por la ley de Leibniz ${ }^{15}$ :

(3) Las sensaciones y sus propiedades son distintos a los estados cerebrales y sus propiedades. (Churchland, 1985, p. 23)

Según Churchland, el problema del argumento presentado es la falta de univocidad (necesaria para la validez del argumento de Jackson) en el sentido de saber acerca de (know about) en la premisa (1) y en la premisa (2). A su juicio:

[...] el tipo de conocimiento adherido en la premisa (1) parece ser claramente diferente del adherido en la premisa (2). El conocimiento en (1) parece ser un asunto de haber dominado un set de oraciones o proposiciones, el tipo que uno encuentra escrito en textos de neurociencias; mientras que el conocimiento en (2) parece ser un asunto de tener una representación de la rojez en algún medio de representación pre-lingüístico o sub-lingüístico para variables sensoriales, o un asunto de ser capaz de hacer ciertas discriminaciones sensoriales, o algo en esas líneas. (Churchland, 1985, p. 23)

De modo que en el caso de la premisa (1), el tipo de conocimiento es por descripción (el dominio de un set de oraciones o proposiciones), mientras que en el caso de la premisa (2), como el mismo Churchland menciona, el tipo de conocimiento es por familiaridad. "La diferencia está en la manera de conocer, no en la naturaleza de la cosa conocida." (Churchland, 1985, p. 24) La diferencia está, en otras palabras, entre el conocimiento que se adquiere a partir de la neurociencia y la física (que obtiene Mary antes de ser liberada) y el tipo de conocimiento que tiene una persona al ver un tomate rojo y que no tiene conocimiento por descripción a partir de la neurociencia y la física (experiencia o vivencia). Siguiendo a Churchland y aplicando dicha distinción al argumento, entonces tenemos lo siguiente:

(a) Mary ha dominado el set completo de oraciones y proposiciones sobre los estados cerebrales de las personas.

(b) Mary no tiene una representación de la rojez en su medio de representación pre- lingüístico para las sensaciones variables.

Entonces, por la ley de Leibniz:

(c) La sensación de rojez es distinta a algún estado cerebral. (Churchland, 1985, p. 24)

\footnotetext{
15 La ley de Leibniz, o principio de identidad de los indiscernibles, establece que, si dos objetos presentan todas sus propiedades en común, entonces ambos son idénticos (Quintero, 2007, p. 8).
} 
En otras palabras, según (a) Mary domina completamente la neurociencia de la visión, pero según (b) no ha tenido una representación de rojez al no experimentar color alguno. Hasta aquí, sostiene Churchland, el fisicalismo estaría de acuerdo. No obstante, la conclusión (c) es precisamente lo que refuta al fisicalismo y le otorgaría razón al argumento de Jackson. Sin embargo, un fisicalista puede sostener que la sensación de rojez no es necesariamente algo más allá de lo físico; es decir, el tener una sensación de rojez, y por tanto aprender de ella (en el sentido de conocimiento por familiaridad), puede tener independencia de una teoría científica que no la explique, sin dejar de ser un evento físico.

\section{b) Horgan: la falacia de Jackson.}

Al igual que Jackson, Horgan considera que los qualia sí existen; sin embargo, a diferencia de Jackson, sostiene que sí son eficaces causalmente e incluso que son propiedades físicas. También, dice, está preparado para aceptar que nosotros no podemos conocer las experiencias de color rojo y $_{1}$ rojo $_{2}$ de Fred, aún sabiendo todos los procesos visuales que se dan en su organismo desde la neurofisiología. También puede aceptar que Mary no puede cónocer el cómo es ver un tomate maduro sin experimentarlo. No obstante, considera que el argumento de Jackson es falaz: "Lo que quiero cuestionar es la suposición de Jackson de que una explicación física adecuada y completa de los procesos visuales de una criatura nos otorguen una completa información física sobre esos procesos." (Horgan, 1984, p. 149)

Esta aseveración puede entenderse de dos modos: en primer lugar, como una tautología, ya que una información física es solamente la que entrega una explicación teoréticamente adecuada. En este sentido, sostener que una explicación física adecuada y completa de los procesos visuales de Fred, por ejemplo, nos otorga una completa información física de dichos procesos, es equivalente a sostener que $A=A$ : la explicación física completa y adecuada de los procesos visuales de Fred no es más que una explicación física completa y adecuada de los procesos visuales de Fred. En segundo lugar, se puede entender como una suposición que un fisicalista puede y debe refutar. Específicamente, Horgan distingue dos nociones de información física:

Digamos que $S$ expresa una información explícitamente física sólo en el caso en que S pertenezca a, o se sigua de, una explicación física teoréticamente adecuada para dichos procesos. $\mathrm{Y}$ digamos que $\mathrm{S}$ expresa información ontológicamente física sólo en caso de (i) todas las entidades refieran a o estén cuantificadas en $\mathrm{S}$ son propiedades físicas, y (ii) todas las relaciones expresadas y relacionadas por los predicados en $\mathrm{S}$ son propiedades y relaciones físicas. (Horgan, 1984, p. 150) 
De modo que una información explícitamente física es expresada mediante un lenguaje manifiestamente físico, mientras que la información ontológicamente física es expresada mediante lenguajes de tipo mental. Para Horgan, Jackson comete un sutil error entre estos dos sentidos de información física, por ejemplo: “Aunque Mary, antes de su primera experiencia de color, tenía un completo stock de información explícitamente física sobre los procesos visuales humanos, es ilegítimo inferir de eso que tiene un completo stock de información ontológicamente física." (Horgan, 1984, p. 151) En otras palabras, Mary, antes de su experiencia con los colores, tenía toda la información explícitamente física por haber sobre la neurofisiología de la visión y la física sobre los colores: es decir, tenía una explicación física completa y adecuada de los procesos de la visión en humanos que le otorgaba una explicación física completa y adecuada de los procesos de la visión en humanos. Pero Mary no tiene la información ontológicamente física antes de su experiencia con los colores, sino sólo explicaciones en el lenguaje teorético de la ciencia física y la neurofisiología. Esta distinción entre los sentidos de información física permiten sostener que lo que Mary no sabía no era información no- física, sino absolutamente física (ya que Horgan sostiene que los qualia son propiedades físicas): entonces, el fisicalismo es verdadero.

\section{c) Papinau: qualia como conceptos epifenoménicos.}

Papineau refiere al argumento del conocimiento de Jackson para demostrar la existencia de conceptos fenoménicos (Papineau, 2002, p. 50). Sostiene, junto a Jackson, que existe una verdadera diferencia entre el antes y el después de que Mary experimentara los colores. Esta diferencia en Mary (a nivel conceptual, dirá Papineau) no es aceptada por todos los fisicalistas. Las respuestas materialistas que no aceptan esta diferencia las denomina como respuestas deflacionarias. La primera respuesta deflacionaria niega que Mary tenga algún cambio sustancial entre antes y después de experimentar el color. La segunda respuesta deflacionaria es la llamada ability hypothesis, la cual sostiene que Mary sí tiene cambios significativos cuando experimenta el color; no obstante, afirma que este cambio involucra su adquisición de nuevas capacidades (abilities) y no nuevos conceptos.

La postura de Papineau acepta la segunda respuesta deflacionaria, pero es inflacionaria conceptualmente, es decir, acepta que Mary adquiere no sólo nuevas capacidades ${ }^{16}$ sino también nuevos conceptos. Los conceptos “[...] son elementos

\footnotetext{
${ }^{16}$ Estas nuevas capacidades, según Papineau, son la capacidad de re-crear imaginativamente y la clasificación introspectiva. La primera corresponde a que Mary, antes de ser liberada y experimentar el tomate rojo, no podía re-crear imaginativamente la
} 
que hacen una contribución sistemática a las condiciones de verdad de los pensamientos en los que entran." (Papineau, 2002, p. 59) La diferencia está en que "En el nivel de referencia, Mary puede seguir pensando sobre algunas propiedades como siempre. Pero en el nivel de la sensación, sus nuevos poderes imaginativos e introspectivos generan una nueva manera de pensar aquellas propiedades." (Papineau, 2002, p. 63) Por ello es que estos conceptos fenoménicos, los qualia, pueden referir perfectamente a propiedades físicas, siempre recordando que para Papineau sólo hay una sustancia física pero un dualismo conceptual. Entonces, el fisicalismo es verdadero.

\section{Fisicalismo reductivista y qualia.}

Respondiendo a la pregunta inicial: ¿constituye el argumento del conocimiento de Frank Jackson una crítica demoledora al fisicalismo reductivo? Mi respuesta es no: el argumento del conocimiento no es una crítica demoledora o un knock out para el fisicalismo reductivo. Si se entiende el fisicalismo reductivo como la doctrina que sostiene que todo es físico y que todo es reducible a lo físico, donde toda propiedad o evento mental $M$ es reductible a alguna propiedad o evento físico $P$, entonces es necesario que los qualia sean propiedades o eventos físicos y que sean reductibles, entonces físicamente. Creo que esto es plausible. Pero es necesario tener en consideración que el tipo de reducción presente en el fisicalismo reductivo pretende explicar $M$ identificándolo con P: en otras palabras, el dolor es la activación de las fibras-C.

La pregunta ante esto es: ¿logra dicha identificación explicar M? Al parecer, una reducción de este tipo parece afectar ontológicamente a $M$, aun cuando $M$ sea $P$ : "[...] al reducirlo estamos compelidos a socavar su posición como existente genuino." (Kim, 2005, p. 95) Parece que $M$, al reducirse a $P$, pierde su carácter de ser $M$. No obstante, aceptando una posición fisicalista con todas sus consecuencias, deberíamos considerar que reducir $\mathrm{M}$ a $\mathrm{P}$ no es socavar el estatuto ontológico de $\mathrm{M}$, sino dar cuenta de su verdadero estatuto, es decir, el dolor no es nada más allá ni por encima de la activación de las fibras- $C$. Sin embargo, como sostuvo Horgan, esta identificación sólo da cuenta de una información explícitamente física, de la cual puede dar cuenta total y completamente la neurofisiología. ¿Qué ocurre con la información ontológicamente física? Atendiendo a la respuesta de Papineau, es

experiencia de rojo al ver el tomate; la segunda, cuando Mary es liberada, es capaz de clasificar introspectivamente distintas experiencias de rojo, por ejemplo. Ver Papineau (2002, pp. 56-59) 
factible sostener que aquello que queda fuera de la explicación en lenguaje teoréticamente físico, no es más que un concepto fenoménico que refiere a nada más allá ni por encima de alguna propiedad física. Vale mencionar que una reducción, al estilo de la teoría de la identidad ${ }^{17}$, no elimina la propiedad reducida 0 , al menos, no pretende eliminarla del lenguaje común: solo quiere dar cuenta que el dolor es la activación de las fibras- $\mathrm{C}^{18}$. ¿Qué ocurre con lo que Mary no sabía?, ¿qué ocurre con los qualia?, ¿son estas cualidades sensoriales reductibles físicamente?

\section{a) Qualia, ¿no físicos?}

Como mostré, hay evidencia para sostener que los qualia pueden ser factiblemente propiedades físicas (Papineau, 2002 o bien Horgan, 1984). Este asunto tiene solución atendiendo a la crítica de Horgan y de Churchland: el argumento de Jackson considera como fisicalismo a una información explícitamente física obtenida de la teoría completa y adecuada de la neurofisiología y la ciencia física; sin embargo, el fisicalismo no deja fuera de consideración a la información ontológicamente física (o al menos no debería hacerlo). Esto quiere decir que los qualia se conozcan por la experiencia y no por la teoría, lo que no implica la falsedad del fisicalismo sino que por el contrario, a mi juicio, lo ratifica aún más. Los qualia, como propiedades fenoménicas, dependen ontológicamente de la perspectiva de quien percibe, la calidad de los órganos perceptivos y el ambiente contextual de la percepción de dicho objeto que produce aquél quale. De modo que pueden perfectamente ser el procesamiento de la información en el cerebro de dichas características particulares. ${ }^{19}$

\section{b) Qualia, ¿epifenoménicos?}

Lo que Mary no conocía eran precisamente los qualia, que según el argumento de Jackson, no eran físicos (al quedar fuera de la explicación fisicalista del mundo). Como ya se dijo, esto es una falacia (Horgan, 1984). Pero el argumento de Jackson no solo quiere probar que los qualia son no-físicos, sino también quiere dar cuenta que son causalmente impotentes ${ }^{20}$. Recuérdese que “[...] es posible que ciertas propiedades

\footnotetext{
${ }^{17}$ Independientemente si es una identidad tipo-tipo o identidad de casos (tokens).

${ }^{18}$ En el caso de los seres humanos, o algún grupo particular de humanos estudiados por la neurofisiología, lo cual no implica que lo que llamamos comúnmente como dolor no pueda ser reducido a algún proceso cerebral.

${ }^{19}$ Por esta razón, un quale es una experiencia irrepetible; pero no por ello es una experiencia no-física.

${ }^{20}$ Precisamente el título del artículo es “Qualia epifenoménicos”, (Jackson, 1982, 2003)
} 
de ciertos estados mentales, a saber, aquellas a las que he llamado qualia, son tales que su posesión o su ausencia no constituye ninguna diferencia para el mundo físico." (Jackson, 1982, 2003, p. 104) Al aceptar la existencia de los qualia se les otorga una ontología física y, por tanto, deben ser causalmente eficaces: su posición en el mundo efectivamente hace diferencias. Un qualia puede ser detectable en la conducta a partir de su rol causal (Kim, 2005, p. 172) al momento de realizar ciertas discriminaciones; por ejemplo, en cómo punza el dolor. Si el dolor punza más fuerte, el resultado conductual es una respuesta evasiva directamente proporcional al qualia específico.

\section{c) ¿Es el argumento de Jackson un knock out al fisicalismo reductivista?}

Finalmente, considero que el argumento de Jackson no constituye un knock-out al fisicalismo reductivo ( $n$ i al fisicalismo en general). Las razones principales son: por un lado, tomo partido por la crítica de Horgan (1984) donde la información física del fisicalismo, que el argumento de Jackson considera como el fisicalismo, dentro de la cual no entran los qualia, es en realidad una información explícitamente física (en un lenguaje teorético de la neurofisiología y la ciencia física). Los qualia quedan fuera de dicha explicación, pero el fisicalismo no niega una información ontológicamente física (en un lenguaje, quizás, mental, por ejemplo) en el cual sí entran los qualia. De modo que si los qualia entran en la explicación física del mundo, entonces son físicos y causalmente eficaces. En este sentido, los qualia no suponen problema alguno para el fisicalismo reductivo: son físicamente reductibles, pero sólo pueden conocerse desde la experiencia. De modo que un correcto fisicalismo reductivo considera tanto la información explícitamente física como la información ontológicamente física.

\section{Conclusión}

El argumento del conocimiento de Frank Jackson fue planteado para defender la existencia de qualia epifenoménicos no-físicos. Las dos versiones del argumento dadas por Jackson, tanto el caso de Fred como el caso de Mary, sostienen que el fisicalismo es falso porque una explicación física completa y adecuada de la neurofisiología de la visión y la física no dan cuenta de las cualidades sensoriales o qualia. De modo que los qualia son no-físicos y el fisicalismo es falso.

Como he intentado mostrar, el argumento de Jackson es inadecuado: por un lado, es una falacia (Horgan, 1984) al confundir dos sentidos distintos de información física: no distingue entre la información explícitamente física en lenguaje teorético de las ciencias y la información ontológicamente física que puede tener un lenguaje, por ejemplo, mentalista. Por otro lado, no es lo mismo saber acerca de (know about) 
procesos cerebrales y sus propiedades, que saber acerca de sensaciones y sus propiedades (Churchland, 1985). Además, los qualia pueden ser perfectamente conceptos fenoménicos que refieren siempre a propiedades físicas, ya que el único mundo existente es el físico (Papineau, 2002). De modo que reducir $M$ a $P$ no significa dejar los qualia fuera de dicha explicación ya que son parte de $\mathrm{P}$.

\section{Referencias}

Alter, T. (2005). The knowledge argument against physicalism. Encyclopedia of Philosophy. Recuperado de http://www.iep.utm.edu/know-arg

Campbell, K. (1987). Cuerpo y mente. México: UNAM.

Churchland, P. (1985). Reduction, qualia, and the direct introspection of brain states. The journal of philosophy, 1(82), 8-28.

Churchland, P. (1989). Knowing qualia: a reply to Jackson. En P. Churchland (1989) Neurocomputational perspective (pp. 67-76). MIT press.

Churchland, P. (2010). Materia y Conciencia. Barcelona: Gedisa.

Horgan, T. (1984). Jackson on physical information and qualia. The philosophical quarterly, 34(135), 147-152.

Jackson, F. (1982). Qualia epifenoménicos. En E. Hansberg (2003) La naturaleza de la experiencia (pp. 111-118). México: Instituto de Investigaciones Filosóficas UNAM.

Jackson, F. (1986). Lo que María no sabía. En O. E. Handsberg (2003). La naturaleza de la experiencia (pp. 95-110). México: Instituto de Investigaciones Filosóficas UNAM.

Jackson, F. (1998). Postscript on qualia. En F. Jackson, Mind, method, and conditional. Selected Essays (pp. 76-79). London: Routledge.

Kim, J. (2005). Physicalism, or something near enough. New Jersey: Princeton University Press.

Kim, J. (2008). Reduction and reductive explanation: Is one possible without the other? En K. Hohwy, Being Reduced (pp. 93-114). Oxford: Oxford University Press.

Moya, C. (2004). Filosofía de la mente. Valencia: PUV.

Papinau, D. (2002). Thinking about consciousness. Oxford: Clarendon Press. 
Place, U. (1956). Is consciousness a brain process? British Journal of Psychology (47), 44-50.

Quinteros, C. (2007). El problema de los indiscernibles. Revista Colombiana de Filosofía de la Ciencia (VIII, 16-17), 7-19.

Stoljar, D. (2009). Physicalism. Stanford Encyclopedia of Philosophy.

Stoljar, D. (2010). Physicalism. London: Routledge.

\section{Agustín Fernando Lavoz Torres}

Es Magíster en Filosofía, Licenciado en Filosofía y Bachiller en Filosofía y Humanidades, titulado de la Universidad Alberto Hurtado. Sus áreas de investigación son la filosofía antigua greco-romana, la filosofía de la mente y la fenomenología alemana. Profesor de pregrado entre 2013 y 2016 en la Universidad Alberto Hurtado en filosofía antigua, moderna y medieval. Desde 2017 es docente del programa semipresencial del Magíster en Coaching Organizacional de la UNIACC donde imparte el curso El arte del coaching con la categoría de titular desde 2019. Además, es Docente Jornada como Apoyo Académico para el Área de Coaching de la Facultad de Administración en UNIACC. 